\title{
DOUBLE INTENSITY INJECTION FOR ANTIPROTON PRODUCTION
}

\author{
K.Y. Ng \\ Fermi National Accelerator Laboratory,* \\ P.O. Box 500, Batavia, IL 60510
}

(August 2002)

\begin{abstract}
The linear density of protons injected into the Fermilab Main Injector is doubled for antiproton production using barrier waves. Adiabatic capture into 53- $\mathrm{MHz}$ buckets can be performed in about $10 \mathrm{~ms}$ with barrier waves on both sides to prevent excessive phase drift outside one booster-batch length. Simulation shows that although the injected protons are captured into $90 \mathrm{rf}$ buckets, the amount of loss in the extra 6 buckets on either sides totals to only $0.51 \%$.
\end{abstract}

${ }^{*}$ Operated by the Universities Research Association, Inc., under contract with the U.S. Department of Energy. 


\section{INTRODUCTION}

A way to increase the luminosity of the Fermilab Tevatron during Run IIa [1] is to increase the number protons delivered to the target for antiproton production. In Ref. [2], a method to inject continuously 12 booster batches into the Main Injector is described in detail. The injection will fill 6/7 of the Main Injector with double intensity, for both antiproton production and the Numi neutrino project in Run IIb [3]. In this paper, we address the special case of filling only one booster-batch length of the Main Injector with double intensity in Run IIa. The problem we are facing is to limit the length of the final proton batch to within a booster-batch length, which is the circumferential length of the antiproton accumulator. Otherwise, losses will occur either at the Main Injector or the antiproton production area. With reasonable sizes of the barrier waves, simulations show that we are able to restrict the double-density protons to $5.9 \%$ longer than the booster-batch length before adiabatic capture. After adiabatic capture with 53-MHz rf, 90 buckets are occupied. The protons in the extra 6 buckets at the sides will be lost eventually. However, these amount to only $0.51 \%$ of the total proton batch. Some injection parameters of the Main Injector are listed in Table I.

Table I: Some injection parameters of the Fermilab Main Injector.

\begin{tabular}{lr}
\hline \hline Nominal total energy $E(\mathrm{GeV})$ & 8.9383 \\
Relativistic $\gamma / \beta$ & $9.326 / 0.99448$ \\
Transition gamma & 21.8 \\
Slip factor $\eta$ & -0.008915 \\
Revolution period $T_{0}(\mu \mathrm{s})$ & 11.1339 \\
Length of booster batch $T_{b}=T_{0} / 7(\mu \mathrm{s})$ & 1.5906 \\
Booster repetition rate $(\mathrm{Hz})$ & 15 \\
\hline \hline
\end{tabular}

In Sec. II, the injection method using a moving barrier is revisited. The simulation is described in Sec. III and the adiabatic capture in Sec. IV. Some comments about beam loading are addressed in Sec. V. A conclusion is made in Sec. VI. 


\section{INJECTION METHOD}

Let us start with a booster batch of protons of length $T_{b}$ and full fractional momentum spread $\Delta$. This batch, denoted by 1 in Fig. 1(a), is injected into the Main Injector at a negative momentum offset, so that the highest fractional momentum offset ${ }^{\dagger}$ is $\delta_{i 1}$ and the lowest fractional momentum offset is $\delta_{i 2}=\delta_{i 1}-\Delta$. The longitudinal position of the injection

(a)
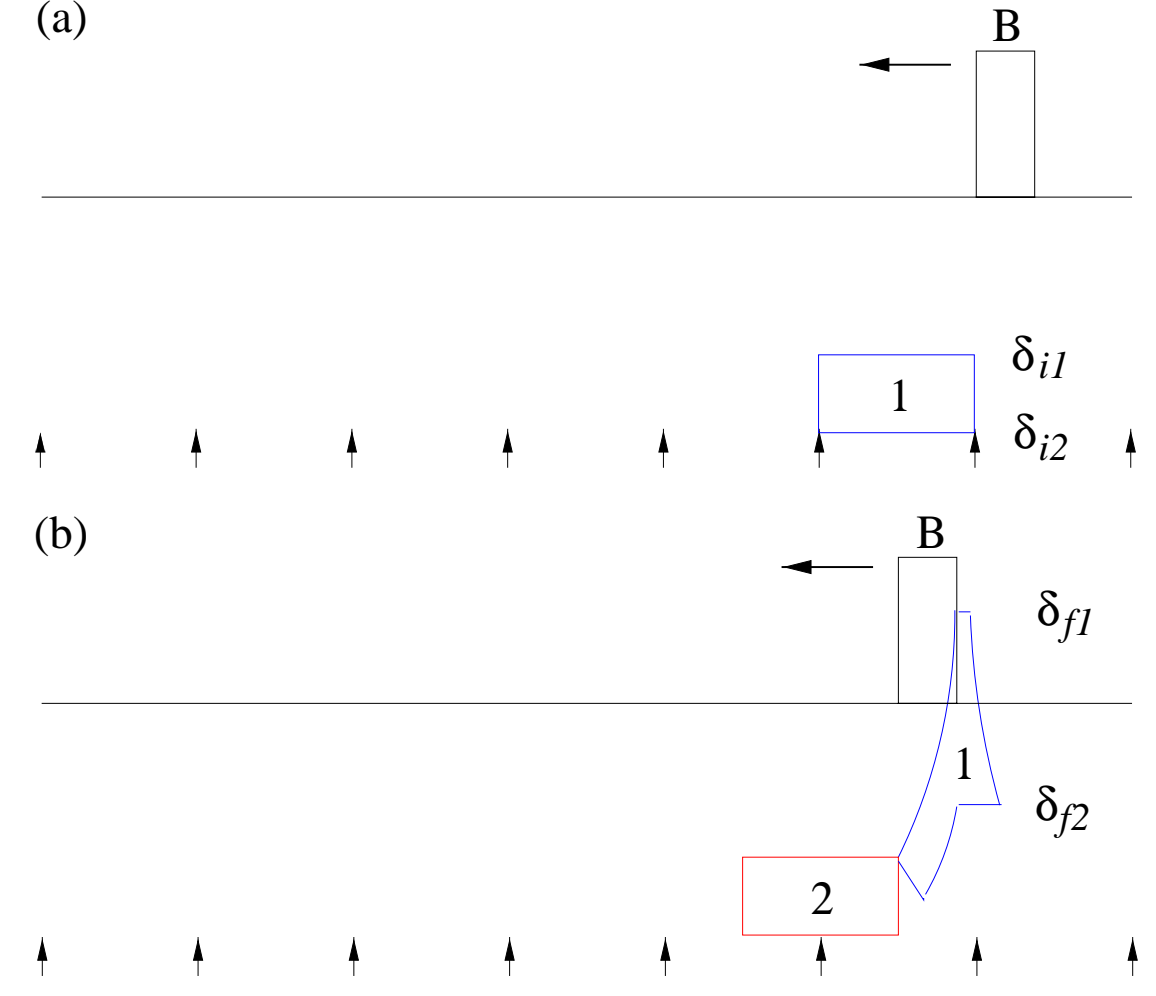

Figure 1: (color) (a) A booster batch with its head at the left (marked 1) is injected at negative momentum offset with the right edge just touching a left-moving barrier (marked B). The vertical arrows are spaced one booster-batch length $T_{b}$. (b) After a booster cycle, the barrier moves by half a booster-batch length while all particles in batch 1 drift past the left edge of the barrier. After exiting the barrier, the batch has maximum and minimum momenta, $\delta_{f 1}$ and $\delta_{f 2}$, symmetric about the on-momentum particles. A second booster batch (marked 2) is now injected.

is so chosen that the right side of the batch just touches the left side of a square barrier (denoted by B) of width $T_{1}$ and magnitude $V$, which is moving to the left at the speed of $\dot{T}_{2}=T_{b} /\left(2 T_{c}\right)$ where $T_{c}$ is the booster-cycle time. The momentum offset of the batch

\footnotetext{
${ }^{\dagger}$ We denote by the subscript 1 the particle at the upper right corner of the batch and 2 the particle at the lower right corner of the batch
} 
injection is so chosen that the proton of highest energy on the left side of the batch will drift to the right at the speed of $\frac{1}{2} T_{b}$ per booster cycle or $-\dot{T}_{2}$. Thus, after one booster cycle, another batch marked 2 in Fig. 1(b) can be injected again with its right edge touching the left side of the barrier. In other words, at every booster cycle, a new booster batch can be injected with the injection point moved half a booster-batch length to the left. The linear beam density can therefore be doubled.

The only parameter here is the size of the barrier, which is so chosen that after the booster batch emerges from the barrier, the highest and lowest energies of the batch become symmetric about the nominal energy of the accelerator ring. This is necessary because we need to place a stationary barrier on the right side of the moving barrier in order to limit the longitudinal motion of the protons after passing through the moving barrier so as to guarantee empty spaces along the Main Injector for successive batch transfer from the Booster. The injection method described here just depends on the integrated size of the barrier and is independent of its shape. As a result, we denote the size of the barrier by $V T_{1}$ and barrier of any shape can be used. However, for the ease of derivation, we continue to assume a rectangular barrier. It is easy to show that [2]

$$
V T_{1}=\frac{|\eta|^{3} \beta^{2} E T_{0}}{32 e \dot{T}_{2}^{2}}\left(2 \delta_{i 1}-\Delta\right)^{2}\left(2 \delta_{i 1}-\Delta-\frac{4 \dot{T}_{2}}{|\eta|}\right)\left(\Delta-\frac{2 \dot{T}_{2}}{|\eta|}\right),
$$

where $E=8.9383 \mathrm{GeV}$ is the injection energy of the Main Injector and $\beta c$ is the particle velocity, $T_{0}=11.1339$ is the revolution period, and $\eta=-0.008915$ is the slip parameter.

Obviously, a larger initial fractional momentum spread $\Delta$ of the beam will lead to a larger final fractional momentum spread. However, when the initial fractional momentum spread is large enough, protons with the highest momentum can acquire so much energy from the moving barrier that their drifting speeds to the left exceed the speed of the moving barrier. The result is that these particles will not be able to emerge from the moving barrier and this injection method fails. This happens when

$$
\dot{T}_{2}<|\eta| \delta_{f 1}
$$

where $\delta_{f 1}$ is emerging fractional momentum spread of the protons with initial momentum spread $\delta_{i 1}$. This gives us the critical size of the moving barrier

$$
\left(V T_{1}\right)_{c}=\frac{|\eta| \beta^{2} E T_{0}}{2 e}\left(\frac{2 \dot{T}_{2}}{|\eta|}\right)^{2}
$$


Correspondingly, the maximum allowable initial full fractional momentum spread of the beam is

$$
\Delta_{c}=(\sqrt{2}-1) \frac{2 \dot{T}_{2}}{|\eta|} .
$$

For the Main Injector, these critical numbers are $\left(V T_{1}\right)_{c}=3.1421 \mathrm{kV}-\mu \mathrm{s}$ and $\Delta_{c}=0.001109$. The critical half energy spread of the batch is therefore $\Delta E_{\frac{1}{2} c}=\Delta_{c} \beta^{2} E / 2=4.900 \mathrm{MeV}$.

\section{INJECTION INTO THE MAIN INJECTOR}

We assume that the half energy spread of the proton bunches extracted from the Booster is $\Delta E_{\frac{1}{2} c}=\Delta_{c} \beta^{2} E / 2=4.900 \mathrm{MeV}$, implying that investigation is performed at the critical situation. We believe that such a half energy spread is possible either by lowering the rf voltage of the Booster in the latter half of the acceleration or by a bunch rotation before extraction. Therefore, the moving barrier will be of the critical value of $\left(V T_{1}\right)_{c}=3.1421 \mathrm{kV}-\mu \mathrm{s}$.

In order to have a shorter batch length after injection, we should place the moving barrier initially as close to the stationary barrier as possible. If it is too close, however, the lowest-energy protons, which emerge first from the moving barrier, will reenter the moving barrier after reflecting from the stationary barrier. This will lead to beam loss. An obvious solution is to use a narrower moving barrier or a higher barrier voltage. Since it is reasonable to generate a barrier voltage up to $V=10 \mathrm{kV}$, this voltage will be used in this discussion. The width of the moving barrier is then $T_{1}=0.31421 \mu \mathrm{s}$. Some parameters of the injection are shown in Table II. The size of the stationary barrier also plays a role in the final proton

Table II: Some parameters in the simulations of this injection method from Fermilab Booster to Main Injector.

\begin{tabular}{lr}
\hline \hline Drifting speed of moving barrier $\dot{T}_{2}=T_{b} / T_{c}$ & $1.193 \times 10^{-5}$ \\
Half energy spread $\Delta E_{\frac{1}{2} i}(\mathrm{MeV})$ & 4.900 \\
Injection energy offset & -11.8285 \\
$\quad$ top of batch $\Delta E_{i 1}(\mathrm{MeV})$ & -21.6276 \\
$\quad$ bottom of batch $\Delta E_{i 2}(\mathrm{MeV})$ & \pm 11.8285 \\
Final half energy spread $\Delta E_{f 1, f 2}(\mathrm{MeV})$ & 3.142 \\
Integrated size of moving barrier $(\mathrm{kV}-\mu \mathrm{s})$ & 0.7855 \\
Minimum integrated size of reflection barrier $(\mathrm{kV}-\mu \mathrm{s})$ & \\
\hline \hline
\end{tabular}


batch length, because there will be more penetration into a lower-voltage barrier before reflection takes place. As a result, we also employ $10 \mathrm{kV}$ for this barrier. A barrier width of $T_{s}=0.18 \mu \mathrm{s}$ is used. This stationary barrier is placed with its right edge at the 7 -th booster-batch length of the Main Injector. Initially, the moving barrier is placed with its left edge at the 6.82-th booster-batch length. At this moment, the first booster batch is injected with its right edge also the 6.82-th booster-batch length and its peak energy particles offset by $-11.8285 \mathrm{MeV}$, as illustrated in the top plot of Fig. 2. The number of macroparticles used has been 40000 per booster batch, although only every 60-th particle is plotted in the figure. One booster cycle later, the moving barrier has its left edge moved to the $(6.82-0.50)$-th booster-batch length, and a second booster batch is now injected with its right edge at the same located as illustrated in lower plot of Fig. 2. In the figure, we see that all particles in the first booster batch have just passed the left edge of the moving barrier and part of the batch has been reflected by the stationary barrier. After another booster cycle, as shown in top plot of Fig. 3, although all particles in the second booster batch have just passed the left edge of the moving barrier, however, some particles form a lagging tail. The moving barrier is allowed to move for another 1181 revolution turns ( 0.197 booster cycle). Now the positiveand negative-momentum-spread particles line up on the left side of the proton batch, and the lagging tail disappears, as is illustrated in the lower plot of Fig. 3. So far the time used has been 2.197 booster cycles or $146.5 \mathrm{~ms}$ and the length of the proton batch exceeds one-booster-batch-length by $5.88 \%$. Since the proton linear density tapers at both the left and right edges, the number of protons outside the one-booster-batch length should be much less. Up to now, no particle loss has been registered. However, if the initial position of the left edge of the moving barrier is at more than the 6.82-th booster-batch length, particle loss will begin to occur.

\section{ADIABATIC CAPTURE}

The protons must be captured into $53-\mathrm{MHz}$ buckets before they can be accelerated. During adiabatic capture, beam particles will wander into adjacent rf buckets and eventually lengthen the beam. To prevent this, we retain the stationary barrier and place another one of the opposite sign near the left edge of the beam. The moving barrier is no longer necessary and is turned off.

For adiabatic capture, we set the criterion that the relative change in bucket area $A_{b}$ 

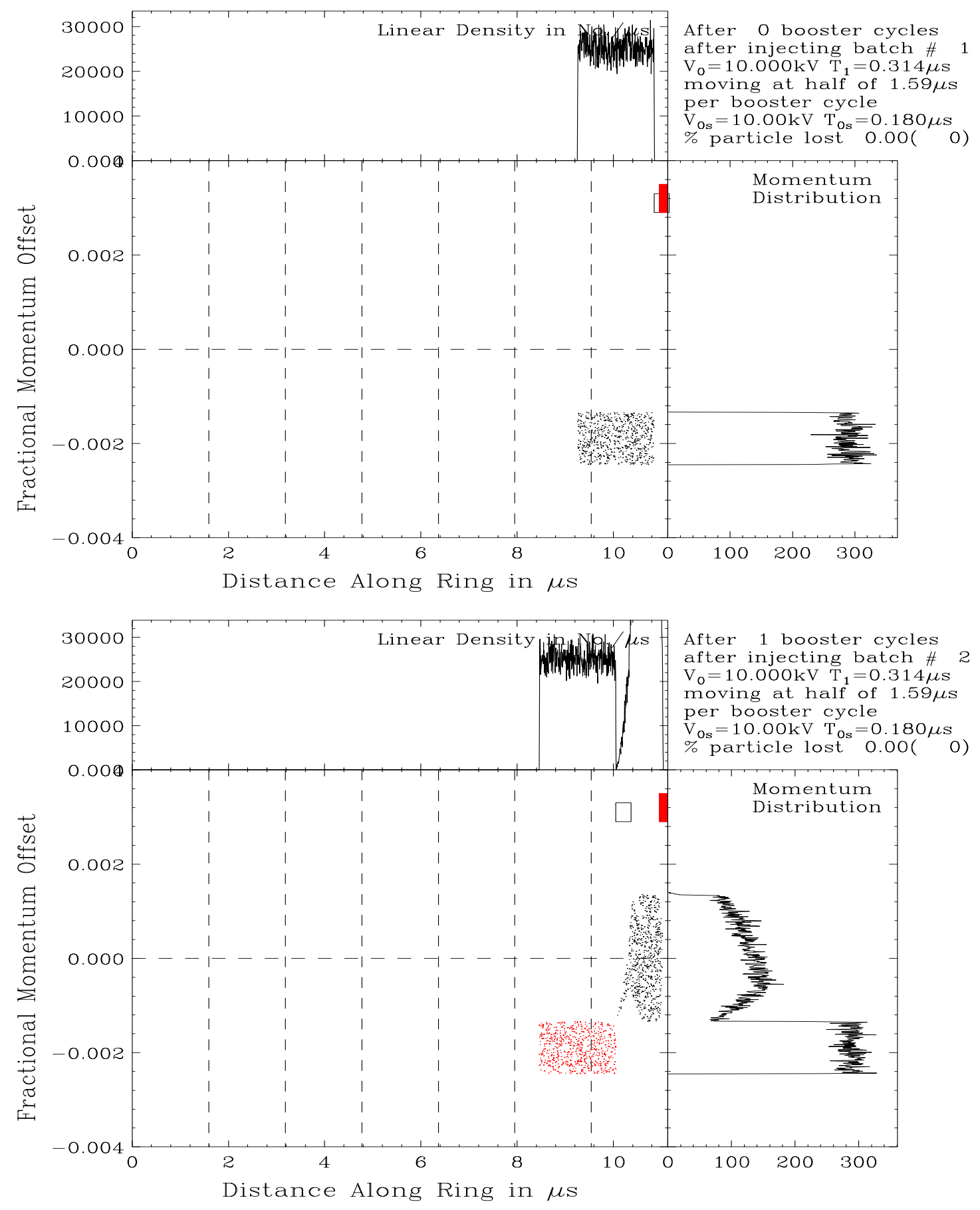

Figure 2: (color) Top: First batch (black) is injected between $5.82 T_{b}$ and $6.82 T_{b}$ with the right side touching the left side of the moving barrier, illustrated as an unfilled box. Bottom: After one booster cycle, all particles in the first batch have just cleared the left side of the moving barrier, which is at $6.0 T_{b}$. The second batch (red) is now injected between $5.32 T_{b}$ and $6.32 T_{b}$. Moving barrier has width $T_{1}=0.31421 \mu$ s and strength $V=10 \mathrm{kV}$. Vertical dashed lines are spaced at $T_{b}$. 

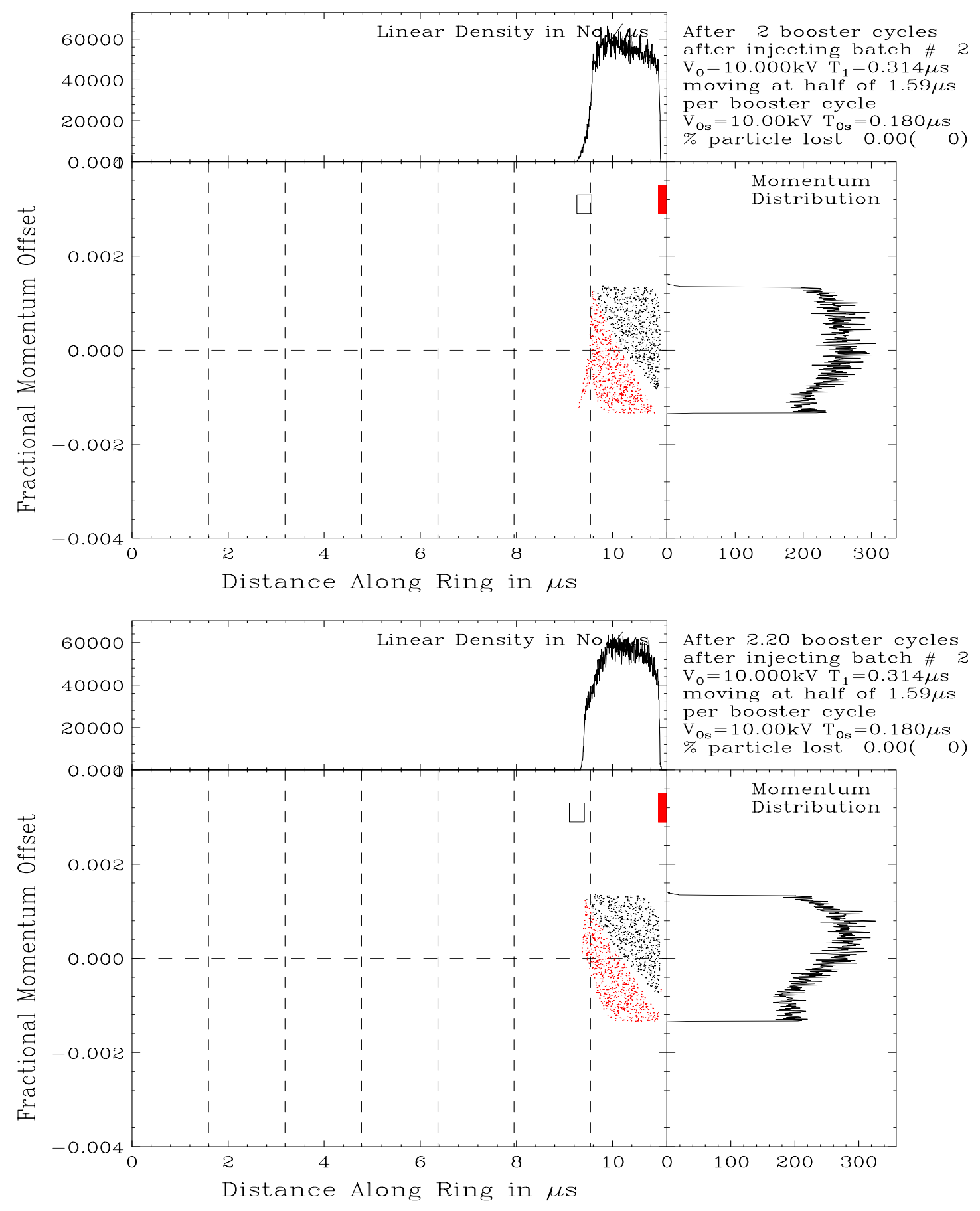

Figure 3: (color) Continuing from top plot of Fig. 2. Top: One booster cycle after the injection of the 2nd batch or 2 booster cycles after the injection of the first batch. A lagging tail of the second batch is seen on the left. Bottom: 0.197 booster cycle later or 2.197 booster cycles after the injection of the first batch. Particles are better aligned at the left edge and adiabatic capture can begin. 
should be much slower than the synchrotron angular frequency $\omega_{s}$, or

$$
\omega_{s}=n \frac{1}{A_{b}} \frac{d A_{b}}{d t}
$$

where $n \gg 1$ is a measure of adiabaticity. Following this, the $53-\mathrm{MHz}$ rf voltage $V_{\mathrm{rf}}$ increases according to

$$
\sqrt{\frac{V_{\mathrm{rf}}(t)}{V_{1}}}=\frac{1}{1-\omega_{s 1} t / n},
$$

where $\omega_{s 1}$ is the angular synchrotron frequency at the initial rf voltage $V_{1}$. As shown in Ref. [2], a rather satisfactory capture can be accomplished from an initial rf voltage $V_{1}=$ $20 \mathrm{kV}$ to a final rf voltage $V_{2}=500 \mathrm{KV}$ in $10.35 \mathrm{~ms}$ with $n=10$. The result of the capture is shown in Fig. 4, where 80000 macroparticles have been used and all of them are plotted. There are all together 90 bunches, whereas a booster batch is only 84-bunch long, implying that 6 of them will be lost eventually. The top and bottom plots of Figure 5 show blowup views of, respectively, the left and right sides of the beam. As expected, we notice that the bunches at both ends are sparsely populated. For example, if we allow the 4 leftmost and the 2 rightmost bunches to be lost eventually, the total loss amounts to only $0.51 \%$.

\section{BEAM LOADING}

Beam loading is important whenever there is a large $53-\mathrm{MHz}$ component $I_{53 \mathrm{MHz}}$ in the beam spectrum. Beam loading will be largest for point bunches, where $I_{53 \mathrm{MHz}} /\left(2 I_{d c}\right)=1$ with $I_{\mathrm{dc}}$ being the dc component. Although the beam is bunched at $53-\mathrm{MHz}$ at transfer from the Booster to the Main Injector, however, because of only barrier waves are used during injection, the beam debunches immediately. Figure 6 shows the evolution of $I_{53 \mathrm{MHz}} /\left(2 I_{d c}\right)=1$ when $8453-\mathrm{MHz}$ bunches enter the moving barrier at injection. Because debunching starts immediately, we see that this ratio drops very rapidly to less than $0.25 \%$ in a few hundred turns, implying that the instantaneous beam loading voltage will be reduced by 400 times as compared to point bunches.

Actually no rf voltage is required in this injection scheme before adiabatic capture. To accomplish this, first, the rf drive of 16 cavities is turned off and shorts are inserted. The Main Injector is presently equipped with fast mechanical shorts, which can be inserted in $100 \mathrm{~ms}$ and removed in $50 \mathrm{~ms}$ [4]. Second, counterphasing is employed for the two remaining cavities to arrive at zero rf voltage with the consideration of the tiny beam loading voltage 


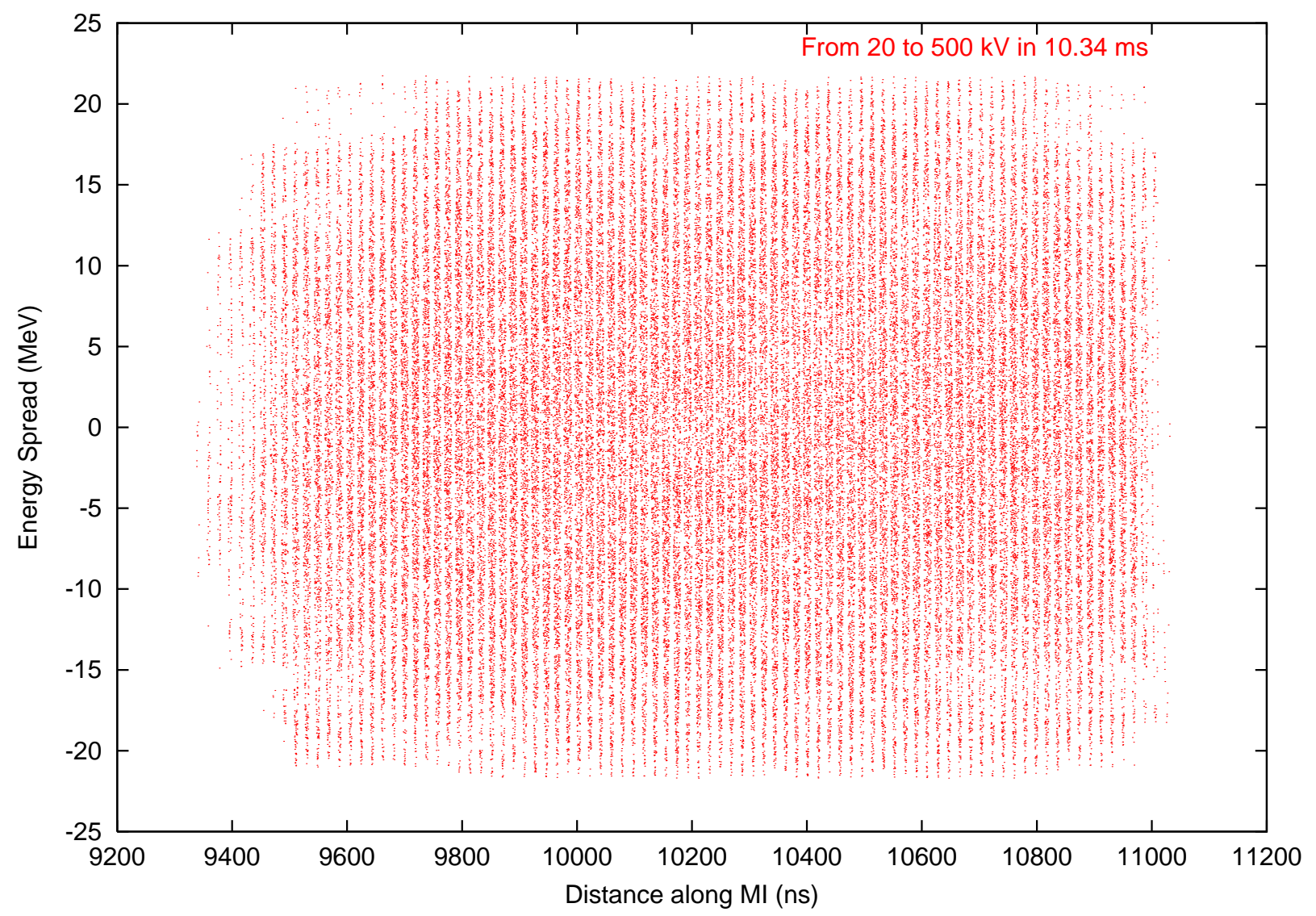

Figure 4: Adiabatic capture of the double-density proton batch by $53-\mathrm{MHz}$ rf. The rf voltage increases from $20 \mathrm{kV}$ to $500 \mathrm{kV}$ in $10.35 \mathrm{~ms}$ according to Eq. (4.2). Two stationary barriers are placed on either side of the beam to prevent longitudinal drifting. The capture results in occupied 90 buckets.

included. Further fine adjustment can be implemented using a fast low-level feedback. The strong beam loading voltage as illustrated in Fig. 6 at the beginning of a batch injection will be reduced by the factor $16 / 18$ as a result of the mechanical shorts, and the rest can be compensated by the fast low-level feedback.

\section{CONCLUSION}

We have devised a scheme to double the linear density of the proton batch in the Main Injector using barrier waves, and the production of antiprotons will be doubled accordingly. The time required comprises $146.5 \mathrm{~ms}$ for injection, $10.35 \mathrm{~ms}$ for adiabatic capture, and 

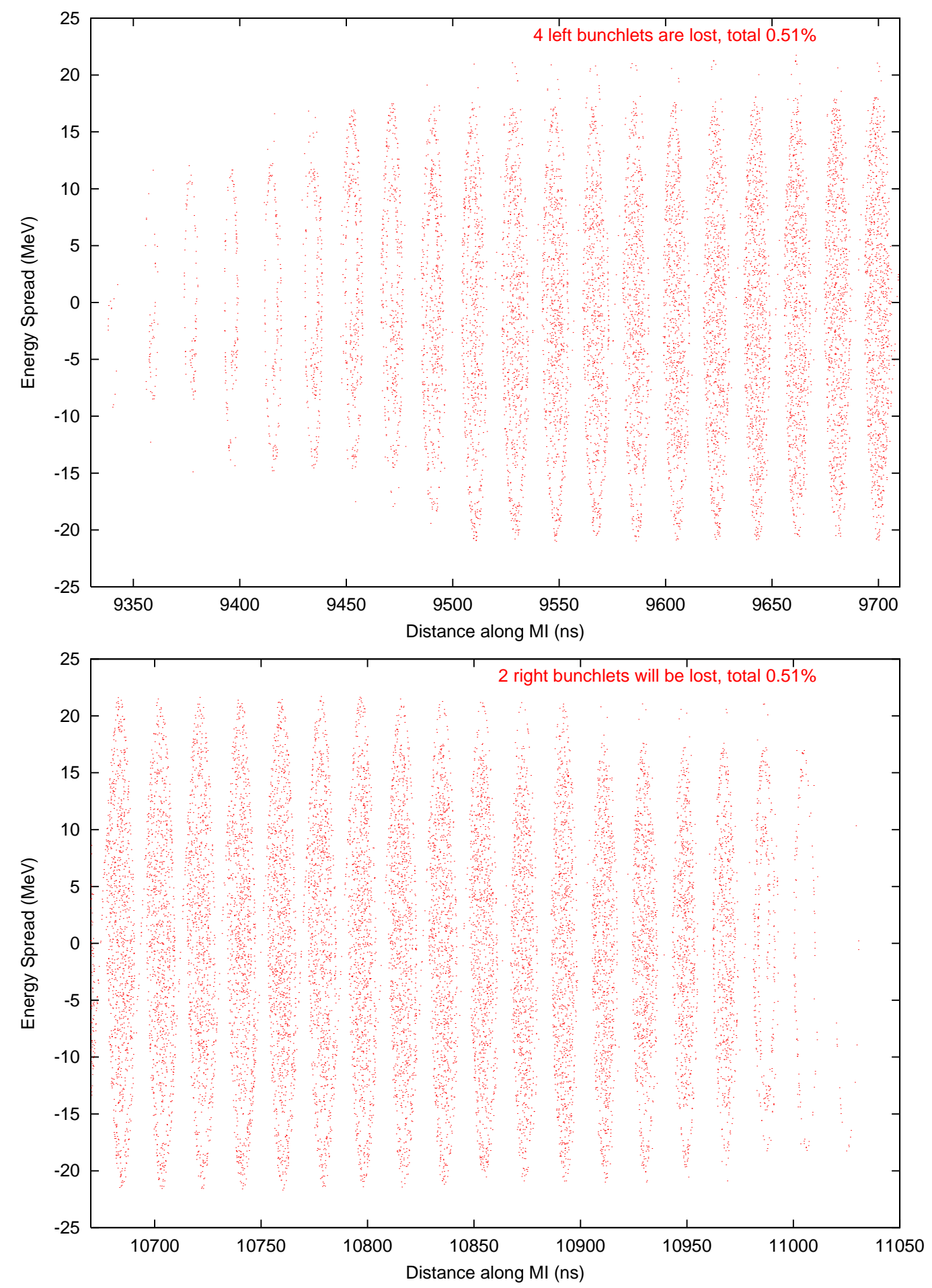

Figure 5: The left (top) and right (bottom) sides of the 90 bunches shown in Fig. 4 are magnified. 4 bunches on the left and 2 bunches on the right will be lost eventually when the center 84 bunches are injected into the antiproton accumulator. The total loss amounts to $0.51 \%$ 


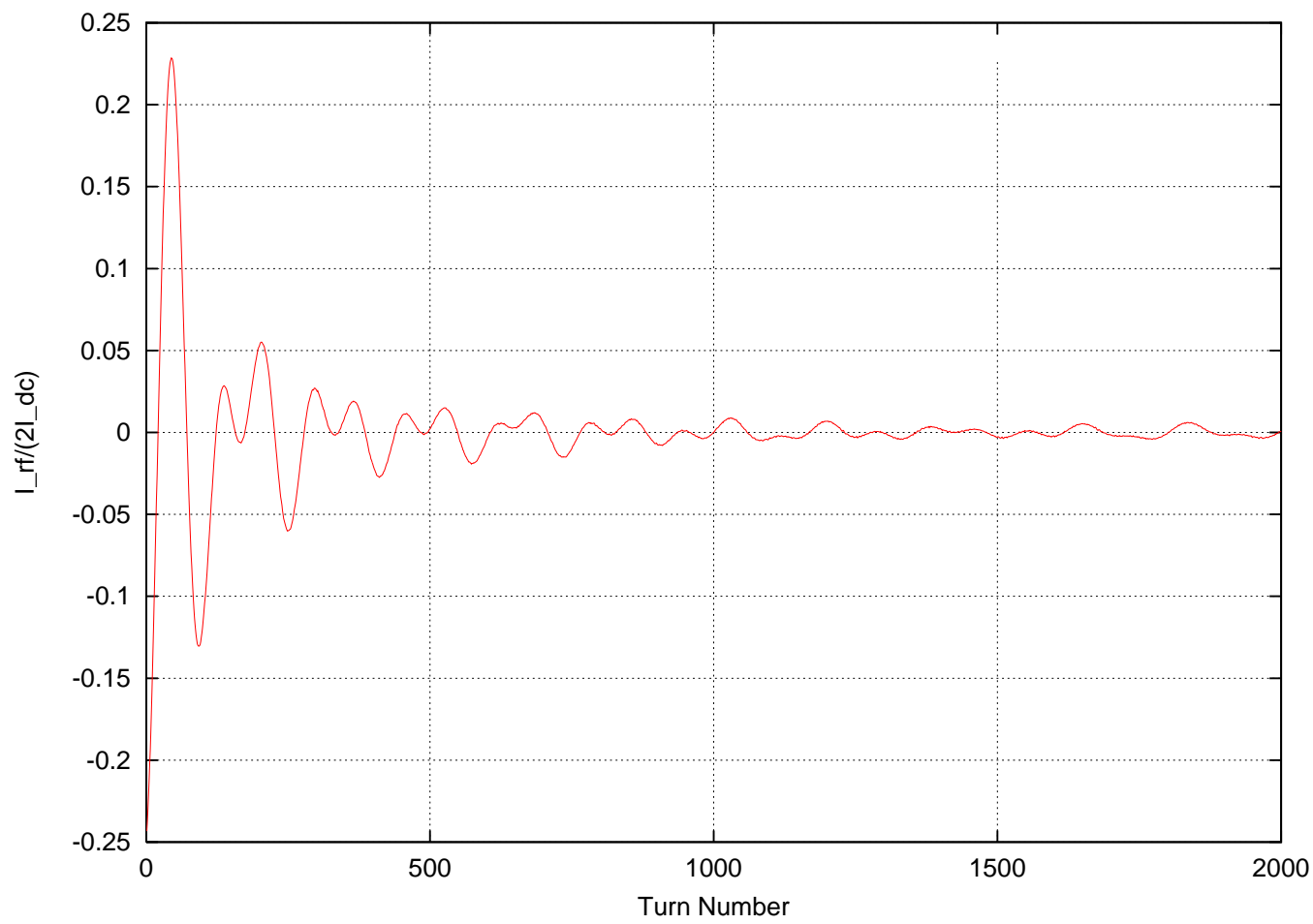

Figure 6: Plot of the ratio of the $53-\mathrm{MHz}$ component of the beam to two times the dc component, $I_{53 \mathrm{MHz}} / I_{\mathrm{dc}}$, as a function of turn number after the injection of the first booster batch consisting of 84 bunches.

$50 \mathrm{~ms}$ for removing the mechanical shorts. The proton batch length has been limited to $5.88 \%$ longer than one booster-batch length with about $0.51 \%$ beam loss when the beam spills into 6 adjacent 53-MHz rf buckets. Further reduction of this type of beam loss is possible by further shortening the length of the proton batch in the Main Injector before adiabatic capture. The obvious method is to employ barriers of higher voltage. In this way, the moving barrier can be placed closer to the stationary barrier and the protons have smaller excursion into the stationary barrier. If a barrier with voltage higher than $10 \mathrm{kV}$ is not available, we can combine the effect of two or more barriers into one.

\section{References}

[1] Run II Handbook, Fermilab Report, http://www-bd.fnal.gov/runII/index.html

[2] K.Y. Ng, Phys. Rev. ST Accel. Beams 5, 061002 (2002), Fermilab Report FN-715, 2002. 
[3] Plan for Tevatron Run IIb, Fermilab Report, http://cosmo.fnal.gov/run2b/Documents/TDR/tdr.pdf

[4] D. Wildman, Transient Beam Loading Reduction during Multi-Batch Coalescing in the Fermilab Main Ring, Proceedings of the 1991 IEEE Particle Accelerator Conference, San Francisco, May 6-9, 1991, p. 410. 